\title{
Near-Field Electrospinning with Close-Loop Motor Control for Manufacturing Polyvinylidene Fluoride Fibers
}

\author{
Cheng-Tang Pan, Kuo-Chang Tsai, ${ }^{*}$ Yun-Ping Sun, ${ }^{1}$ and Chung-Ken Yen \\ Department of Mechanical and Electro-Mechanical Engineering, National Sun Yat-sen University \\ Kaohsiung 80424, Taiwan \\ ${ }^{1}$ Department of Mechanical Engineering, Cheng Shiu University, Kaohsiung 83347, Taiwan
}

(Received September 30, 2016; accepted January 17, 2017)

Keywords: near-field electrospinning (NFES), polyvinylidene fluoride (PVDF), piezoelectric fiber

In this study, we report a close-loop motor control for a cylindrical collector to obtain continuous and uniform polyvinylidene fluoride (PVDF) piezoelectric fiber arrays using near-field electrospinning (NFES). The as-spun PVDF piezoelectric nanofibers were laid on the top of parallel electrodes to generate current and voltage signals by means of mechanical energy of low-frequency vibrations being converting to electrical energy. The PVDF fibers can be used as a flexible sensing device, such as a smart fabric, owing to their excellent voltage response. A PVDF fiber with continuous and uniform diameter is expected to have stable energy conversion for achieving higher electrical current and voltage signals. To ensure the quality of the production process, a closeloop system for motor speed control was designed to improve on the disadvantages of open-loop systems, such as sensitivity to disturbances and inability to compensate for these disturbances automatically. Transient response and steady-state error can be controlled more conveniently and accurately. The experimental scheme was designed using Taguchi Methods. A mixed solution of $18 \mathrm{wt} \%$ PVDF was placed in a metal needle injector and driven by a syringe pump at $0.2,0.4$, and $0.8 \mathrm{ml} / \mathrm{h}$ feeding rates. The needles with $0.2,0.25$, and $0.33 \mathrm{~mm}$ diameters were subjected to $13 \mathrm{k}, 15 \mathrm{k}$, and $17 \mathrm{k}$ high voltages with a gap of $1 \mathrm{~mm}$ between the metal needle and the cylindrical collectors (electrical fields: $E=1.3 \times 10^{7}, 1.5 \times 10^{7}$, and $1.7 \times 10^{7} \mathrm{~V} / \mathrm{m}$, respectively). The metal needle travelled in the axial direction of the cylindrical collectors at 120,160 , and $200 \mathrm{~mm} / \mathrm{min}$, respectively. The cylindrical collector motor operated with six different speeds in the range of 900-1900 rpm. With the motor speed control system, about $I_{p-p}=8.8 \times 10^{-7} \mathrm{~A}\left(I_{\max }=-5.0 \times 10^{-7} \mathrm{~A}\right)$ and $V_{p-p}=0.22 \mathrm{~V}\left(V_{\max }=-0.12 \mathrm{~V}\right)$ were obtained from the PVDF piezoelectric fiber array, which is obviously better than its counterpart of about $I_{p-p}=3.2 \times 10^{-7} \mathrm{~A}\left(I_{\max }=-2.1 \times 10^{-7} \mathrm{~A}\right)$ and $V_{p-p}=0.17$ $\mathrm{V}\left(V_{\max }=-0.10 \mathrm{~V}\right)$ with the open-loop system.

\section{Introduction}

After decades of developments in the miniaturization of portable and wireless devices, new power sources beyond rechargeable batteries have become important topics for current and future stand-alone devices and systems. Specifically, ideal power sources should be scalable for the power demands of various portable devices without the necessity of a recharging process or replacement.

"Corresponding author: e-mail: vongohking@yahoo.com.tw http://dx.doi.org/10.18494/SAM.2017.1533 
Recent work in the field of nanomaterials has shown considerable progress toward self-powered energy sources by scavenging energy from ambient environments, such as mechanical vibration. In particular, the use of piezoelectric generators by nanomaterials as a simple and effective solution for mechanical energy harvesting has attracted considerable attention. ${ }^{(1)}$

In addition, devices such as piezoelectric ${ }^{(2-6)}$ transducers should operate at high-frequency vibrations to create a higher power generation efficiency. These energy harvesters become one of the key factors in these designs. It is known that vibration-based mechanical energy is the most accessible energy source in surroundings. For random vibrations with frequencies from hundreds of $\mathrm{Hz}$ to $\mathrm{kHz}$, the available energy density is within the range of a few hundred microwatts to milliwatts per cubic centimeter. ${ }^{(7,8)}$ Therefore, energy harvesting from this type of vibration offers a great potential for remote/wireless sensing, charging batteries, and powering electronic devices. ${ }^{(9-12)}$ Mechanical energy scavenging from ambient environments is an attractive renewable source of power. Conventional electrospinning processes producing randomly deposited nanofibers have been used in various applications including filtration, texturing, composite reinforcement, and tissue scaffolds. The disorderly behavior of such deposited nanofibers has limited their full potential. ${ }^{(13,14)}$ A direct-write electrospinning technique using near-field electrospinning (NFES) ${ }^{(13,15)}$ has been developed to produce directional controllable depositions of fibers. Previously, Chang et al. reported on nanogenerators based on electrospun polyvinylidene fluoride (PVDF) fibers with high energy conversion efficiency for potential wearable smart clothes to power hand-held electronics by body movements. ${ }^{(16)}$ Here, the piezoelectric actuation characteristics of a single electrospun PVDF fiber were investigated for potential applications in actuators, switches, and artificial muscles. ${ }^{(17)}$ These fibers were made of PVDF with high flexibility, minimizing resistance to external mechanical movements in low-frequency, large-deflection energy scavenging applications. PVDF has good piezoelectric and mechanical properties, exhibits chemical stability and weathering characteristics, ${ }^{(16,18,19)}$ and can be constructed as thin films for sensing and actuation applications. ${ }^{(20-23)}$

In this paper, NFES with close-loop motor control was carried out to fabricate PVDF piezoelectric fibers with continuous and uniform diameters. We developed a close-loop system that was less sensitive to disturbances and changes in the environment to ensure a steady and accurate motor speed. The steady speed of a cylindrical collector resulted in a uniform diameter as well as good quality of the PVDF fibers. The experimental scheme was designed using Taguchi methods to obtain optimal process parameters. Good-quality fibers can be fabricated for a sensing device. In this study, we examined the characteristics of the PVDF fibers by measuring electric properties (voltage and current) and carried out the experiment at a low frequency of $7 \mathrm{~Hz}$ tapping on the PVDF fiber samples. Morphological inspection via scanning electron microscopy (SEM) provided perspective for the quality check of PVDF fibers.

\section{Materials and Methods}

\subsection{Materials preparation}

The $18 \mathrm{wt} \%$ PVDF solution used in the continuous NFES experiments was prepared with a powder of polymer PVDF, acetone, dimethyl sulfoxide, $\left(\mathrm{CH}_{3}\right)_{2} \mathrm{SO}$ (DMSO), and surfactant. The concentration of $18 \mathrm{wt} \%$ was chosen based on the previous study. ${ }^{(24)}$ The PVDF powder was dissolved in acetone. The surfactant changed the surface tension of the solution to make the 
electrospinning go smoothly. The solution was stirred by a magnet and a magnetic stirrer. After the NFES process, the PVDF fibers were deposited on a cylindrical collector. The electric properties of the as-spun fibers were evaluated at low frequency.

\subsection{Equipment system with close-loop motor control}

In the study, we explored NFES to fabricate continuous and uniform piezoelectric PVDF fibers; the equipment system is shown in Fig. 1. In the experiments, the motors of AKD Servo Drives (National Instruments AKD-P00306) and Brushless Servo Motors (National Instruments AKM11B) were chosen for the close-loop system. The AKD WorkBench provided by Kollmorgen, a leading provider of motion systems and components, was used as human-machine interface (HMI) software that allowed us to conveniently tune the parameters of the AKD servo motor system. By a standard Ethernet for Control Automation Technology, called EtherCAT, we connected it to the drive to both view and change drive the parameters of AKD WorkBench easily and quickly. The configuration of the close-loop control system is shown in Fig. 2. With fast control-loop update rates, the closeloop control system not only guaranteed a reliable control performance, but also accommodated changing load conditions.

\subsection{Measurement method}

The experiments were designed by Taguchi Methods using five factors with 6 and 3 levels. There are five parameters, namely, cylindrical collector speed, electrospinning voltage, syringe pump feeding rate, needle moving speed, and needle diameter (see Table 1). The PVDF solution was filled into an injector, which was infused by a syringe pump at $0.2,0.4$, and $0.8 \mathrm{ml} / \mathrm{h}$ rates. The solution was supplied by the syringe pump to the tip of a metal needle where $13 \mathrm{k}, 15 \mathrm{k}$, and $17 \mathrm{k}$ high voltages were applied. The positive side of the high-voltage was applied to the needle (diameters:

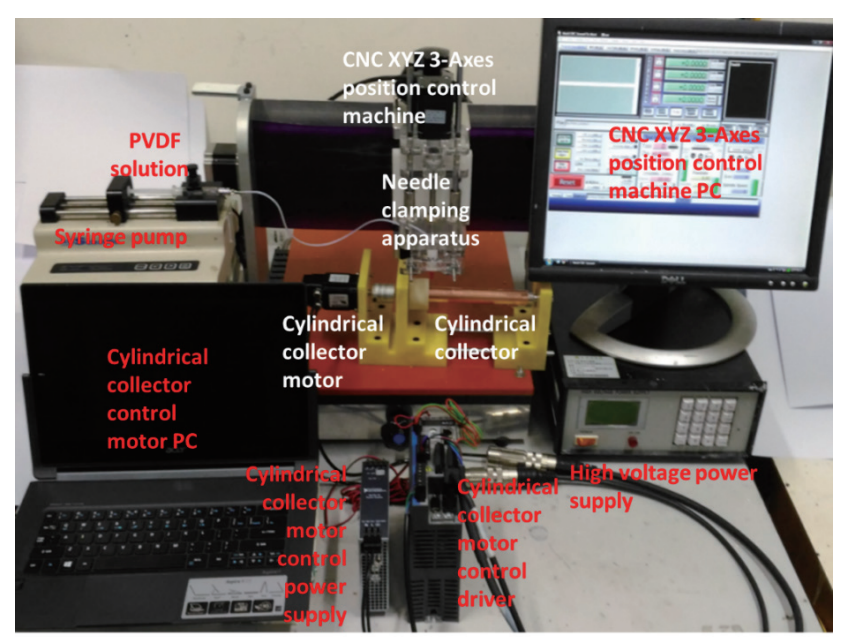

Fig. 1. (Color online) Equipment setup of NFES.

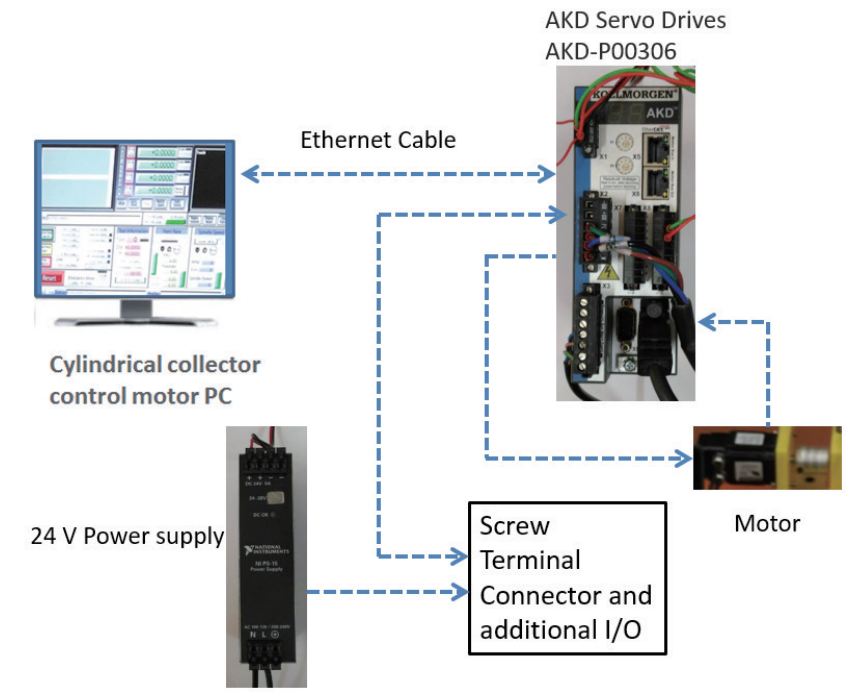

Fig. 2. (Color online) Configuration of the closeloop motor control system. 
Table 1

$\mathrm{L}_{18}\left(6^{1} \times 3^{6}\right)$ orthogonal arrays (five parameters with 6 and 3 levels).

\begin{tabular}{lcccccccc}
\hline Five parameters with 6 and 3 levels & No. & 1 & 2 & 3 & 4 & 5 \\
\hline 1. Clyindrical collector speed & 1 & $900 \mathrm{rpm}$ & 1 & 1 & 1 & 1 & 1 & 1 \\
& 2 & $1100 \mathrm{rpm}$ & 2 & 1 & 2 & 2 & 2 & 2 \\
& 3 & $1300 \mathrm{rpm}$ & 3 & 1 & 3 & 3 & 3 & 3 \\
& 4 & $1500 \mathrm{rpm}$ & 4 & 2 & 1 & 1 & 2 & 2 \\
& 5 & $1700 \mathrm{rpm}$ & 5 & 2 & 2 & 2 & 3 & 3 \\
& 6 & $1900 \mathrm{rpm}$ & 6 & 2 & 3 & 3 & 1 & 1 \\
\hline 2. Electrospinning voltage & 1 & $13 \mathrm{kV}$ & 7 & 3 & 1 & 2 & 1 & 3 \\
& 2 & $15 \mathrm{kV}$ & 8 & 3 & 2 & 3 & 2 & 1 \\
& 3 & $17 \mathrm{kV}$ & 9 & 3 & 3 & 1 & 3 & 2 \\
\hline 3. Syringe pump feeding rate & 1 & $0.2 \mathrm{ml} / \mathrm{h}$ & 10 & 4 & 1 & 3 & 3 & 2 \\
& 2 & $0.4 \mathrm{ml} / \mathrm{h}$ & 11 & 4 & 2 & 1 & 1 & 3 \\
& 3 & $0.8 \mathrm{ml} / \mathrm{h}$ & 12 & 4 & 3 & 2 & 2 & 1 \\
\hline 4. Needle moving speed & 1 & $120 \mathrm{~mm} / \mathrm{min}$ & 13 & 5 & 1 & 2 & 3 & 1 \\
& 2 & $160 \mathrm{~mm} / \mathrm{min}$ & 14 & 5 & 2 & 3 & 1 & 2 \\
& 3 & $200 \mathrm{~mm} / \mathrm{min}$ & 15 & 5 & 3 & 1 & 2 & 3 \\
\hline 5. Needle diameter & 1 & $0.33 \mathrm{~mm}$ & 16 & 6 & 1 & 3 & 2 & 3 \\
& 2 & $0.25 \mathrm{~mm}$ & 17 & 6 & 2 & 1 & 3 & 1 \\
& 3 & $0.2 \mathrm{~mm}$ & 18 & 6 & 3 & 2 & 1 & 2 \\
\hline
\end{tabular}

$0.2,0.25$, and $0.33 \mathrm{~mm}$ ), and the negative side was applied to the cylindrical collector. The gap between the needle tip and cylindrical collector was set at $1 \mathrm{~mm}$. The metal needles with $0.2,0.25$, and $0.33 \mathrm{~mm}$ diameters travelled along the axial direction of the cylindrical collector with speeds of 120,160 , and $200 \mathrm{~mm} / \mathrm{min}$, respectively. The cylindrical collector motor was operated at six different speeds $(900,1100,1300,1500,1700$, and $1900 \mathrm{rpm})$.

The quality of PVDF fibers was evaluated by measuring their electrical properties. By means of the energy harvesting via parallel electrodes with $7 \mathrm{~Hz}$ tapping on the PVDF fibers, we obtained the voltages and currents. By the SEM inspection, we observed the morphology of the fibers to realize the relation between the electrical properties and the morphology.

\section{Results and Discussion}

We analyzed the PVDF fibers with respect to their diameters and electrical properties to evaluate the NFES process with open- and close-loop motor controls. In this study, cylindrical collector motor speed was operated by close-loop control with AKD Servo Drives and Brushless Servo Motors. We used three cylindrical collector speeds, namely, 900, 1500, and $1900 \mathrm{rpm}$, to evaluate the PVDF fibers via voltage and current measurements.

\subsection{Electrical properties test by energy harvesting}

The whole tapping and measuring system is shown in Fig. 3, including equipment for measuring of voltage, strain, and current. The National Instrument cDAQ-9174 is a 4-slot Compact DAQ USB chassis designed for small, portable, and mixed-measurement systems. We combine the cDAQ-9174 with several NI C series I/O modules for voltage and strain measurements. The $\mathrm{CH}$ 


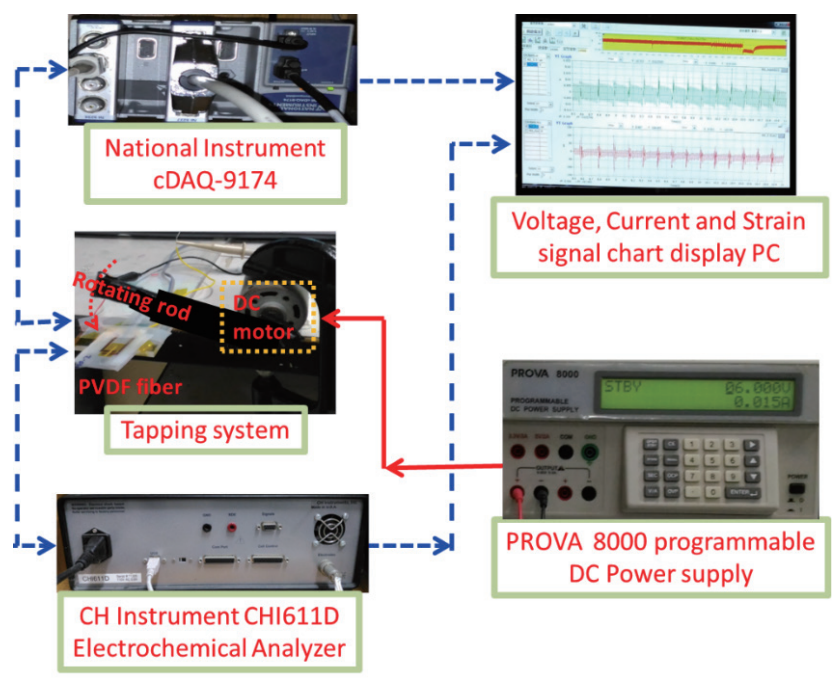

Fig. 3. (Color online) PVDF fiber sample tapping and electric property measurement system.

Instrument CHI611D Electrochemical Analyzer was for current measurement. The PROVA 8000 Programmable DC Power Supply supplied power to the tapping motor.

Figures 4 and 5 show the results of the comparison of current and voltage between the closeloop and open-loop motor control systems. The parameters are those of experiment 2 in Table 1 (cylindrical collector speed of $900 \mathrm{rpm}$, electrical field $E=1.5 \times 10^{7} \mathrm{~V} / \mathrm{m}$, syringe pump feeding rate of $0.4 \mathrm{ml} / \mathrm{h}$, needle moving speed of $160 \mathrm{~mm} / \mathrm{min}$, and needle diameter of $0.25 \mathrm{~mm}$ ).

Figure 4 shows current charts. The tapping test showed a positive current of about $3.8 \times 10^{-7}$ and a negative current of about $-5.0 \times 10^{-7} \mathrm{~A}$ with the close-loop motor control system [see Fig. 4(a)]. In comparison with a positive current about $1.1 \times 10^{-7} \mathrm{~A}$ and negative current about $2.1 \times$ $10^{-7} \mathrm{~A}$ with the open-loop system, the close-loop control achieved very clear improvement. There are three advantages of a close-loop motor control system [Fig. 4(a)] over the open-loop system [Fig. 4(b)]: (1) higher currents $\left(3.8 \times 10^{-7}>1.1 \times 10^{-7} ; 5.0 \times 10^{-7}>2.1 \times 10^{-7}\right.$, only considering magnitude), (2) more uniform in peak values, and (3) more balanced current distributions on both positive and negative sides (the close-loop motor control has better positive/negative balance than the open-loop one).

Figure 5 shows voltage charts. The tapping test showed a positive voltage of about $0.10 \mathrm{~V}$ and a negative voltage of about $-0.12 \mathrm{~V}$ with the close-loop motor control system [see Fig. 5(b)]. In comparison with positive current of about $0.07 \mathrm{~V}$ and negative current about $-0.09 \mathrm{~V}$ with the openloop system, the close-loop control also achieved clear improvement. There are three advantages of the close-loop motor control system [Fig. 5(a)] over the open-loop system [Fig. 5(b)]: (1) higher voltages $(0.10>0.07 ; 0.12>0.09$, only considering magnitude), (2) more uniform peak values, and (3) more balanced voltage distributions on both the positive and negative sides (the close-loop motor control has better positive/negative balance than the open-loop one). However, the improvement was not as large as in the case of current (Fig. 4).

To confirm that the signals came from the piezoelectric property, we added the reverse electric connection (reverse pole) test for proof. The reverse voltage signals of PVDF fibers were obtained at a tapping frequency at $5 \mathrm{~Hz}$. In comparison with the forward electric connecting chart of the 


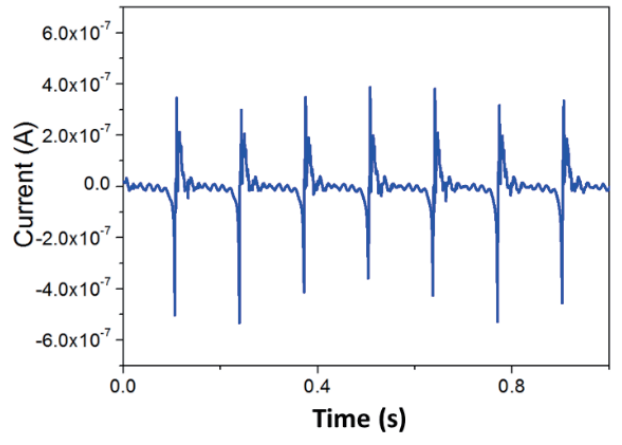

(a)

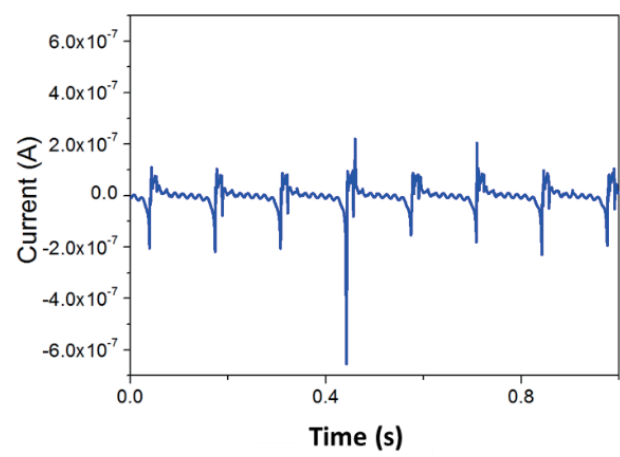

(b)

Fig. 4. (Color online) Carried out with parameters of experiment 2 in Table 1: (a) close-loop control current and (b) open-loop current.

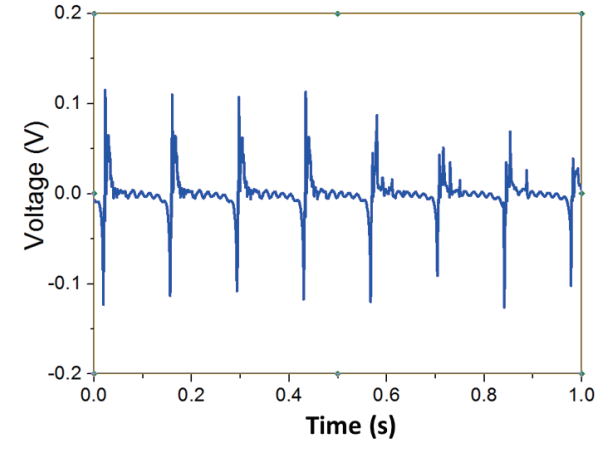

(a)

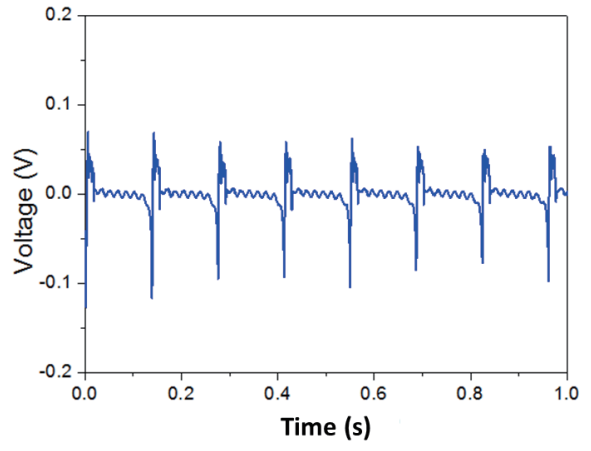

(b)

Fig. 5. (Color online) Carried out with parameters of experiment 2 in Table 1: (a) close-loop control voltage and (b) open-loop voltage.

close-loop motor control in Fig. 6(a), the reverse connection chart in Fig. 6(b) reveals a voltage trend chart with an "upside down pattern". The forward and reverse connections of the open-loop system are compared in Figs. 6(c) and 6(d), respectively. The strain charts of the forward electric connection of the close-loop motor control and open-loop system are shown in Figs. 5(e) and 5(f), respectively.

\subsection{Properties and characteristics of PVDF fiber}

Figure 7(a) shows that the diameters of the PVDF fibers decreased from 33.12 to $13.30 \mu \mathrm{m}$ in the close-loop system and from 33.53 to $14.21 \mu \mathrm{m}$ in the open-loop system with increasing tangential velocity of the collector device from 943 to $1990 \mathrm{~mm} / \mathrm{s}$. At a higher rotational speed of the cylindrical collector, the PVDF fiber received a greater drawing force which resulted in thinner fibers. Figure 7(b) shows the diameters of the PVDF fibers in different electrical fields $(E=1.3 \times$ $10^{7}, 1.5 \times 10^{7}$, and $\left.1.7 \times 10^{7} \mathrm{~V} / \mathrm{m}\right)$. Higher electrostatic fields caused stronger electrostatic forces and resulted in thinner PVDF fibers. The diameter of the PVDF fibers decreased from 15.21 to 


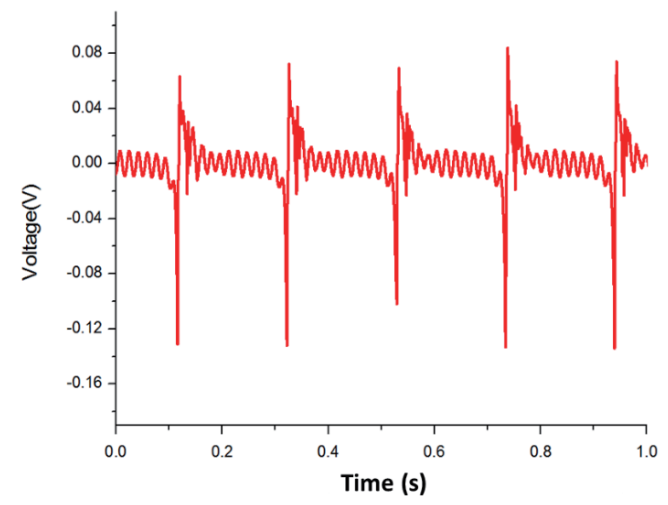

(a)

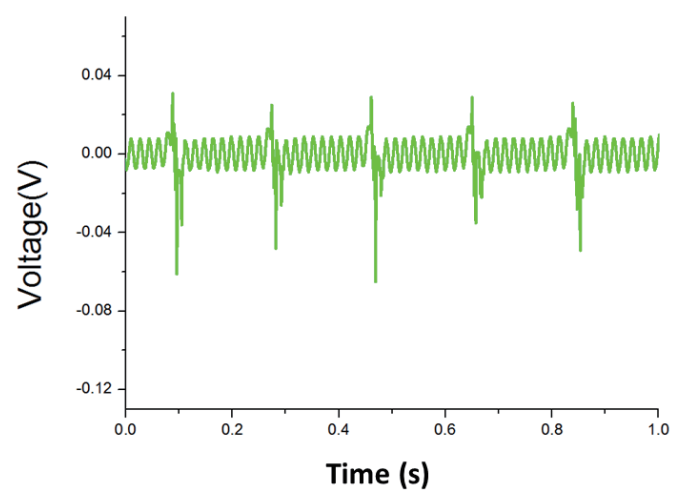

(c)

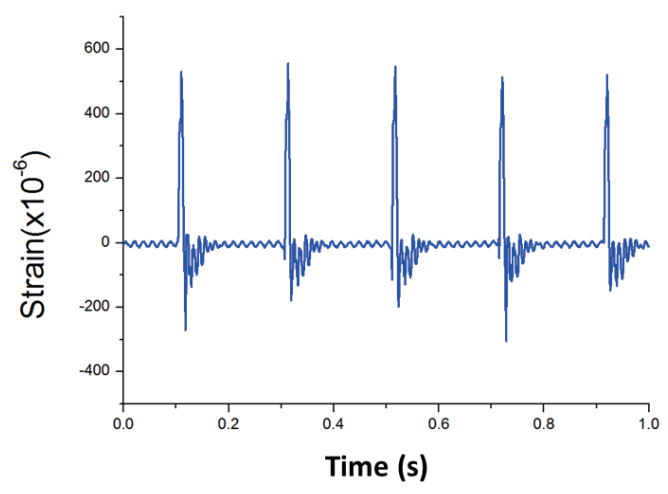

(e)

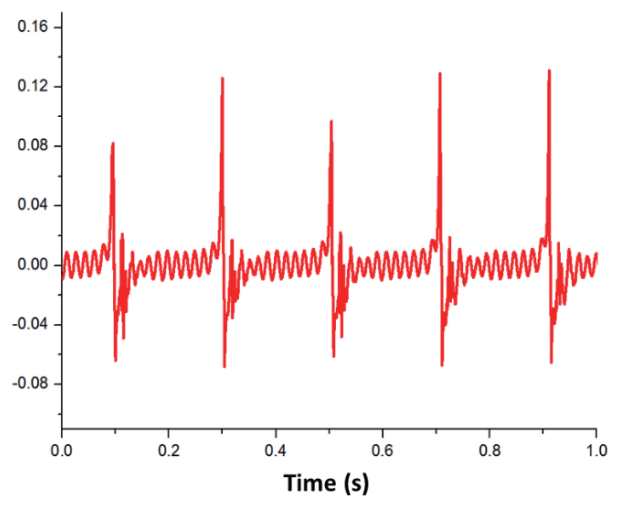

(b)

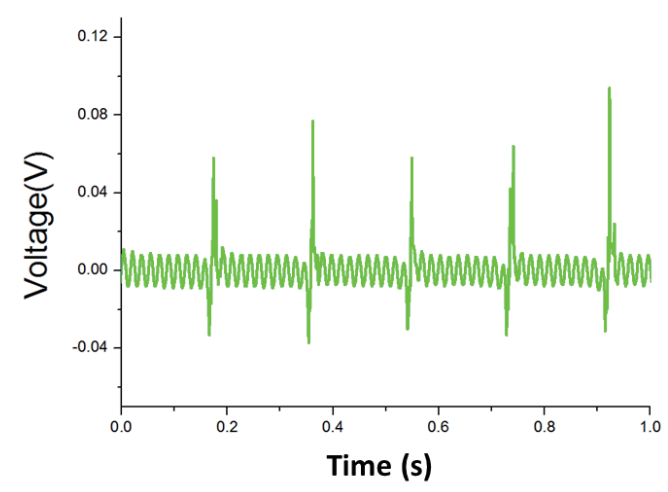

(d)

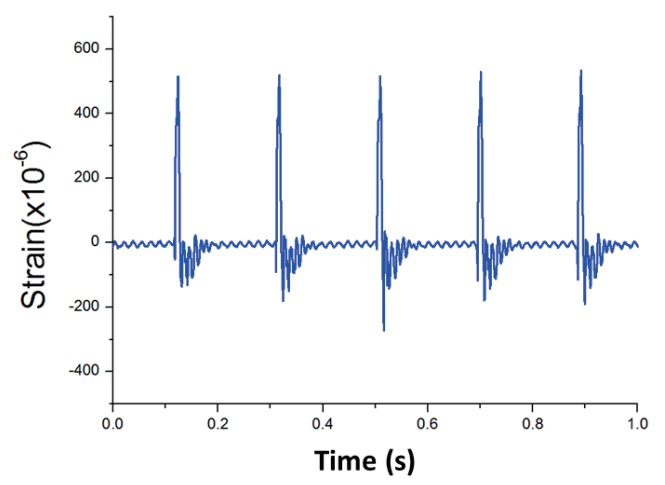

(f)

Fig. 6. (Color online) Voltage charts of (a) forward and (b) reverse electric connections of close-loop motor control. Voltage charts of (c) forward and (d) reverse connections of open-loop system. Strain charts of forward electric connection of (e) close-loop motor control and (f) open-loop system (carried out with parameters of experiment 2 in Table 1).

$12.05 \mu \mathrm{m}$ in the close-loop control system and from 15.97 to $12.91 \mu \mathrm{m}$ in the open-loop control system (with larger fluctuation in the close-loop control) when the electrical field increased from $1.3 \times 10^{7}$ to $1.7 \times 10^{7} \mathrm{~V} / \mathrm{m}$. These diameters decreased with increasing electrical field, but shortcircuiting phenomena occurred when the electrical field was too high. Thus, the electrospinning field had to be controlled to within a suitable voltage range. 
The PVDF powders included $\alpha-, \beta$-, and $\gamma$-phase crystal as determined by X-ray diffraction (XRD). Before NFES processing (polarization processing), the major ingredient was $\alpha$-phase crystals. The $\alpha$-phase crystals did not have piezoelectric properties because the dipole moments of the $\alpha$-phase crystals were not oriented, whereas the dipole moments of the $\beta$-phase crystals were aligned. The NFES converted $\alpha$-phase crystals into $\beta$-phase crystals to confer piezoelectric properties by aligning dipole moments. As shown in Fig. 8, before NFES processing, the major ingredients of the PVDF powders were $\alpha$-phase crystals. After NFES processing, the major ingredients of the PVDF powder were $\beta$-phase crystals. Figure 9 shows the NFES processing with a cylindrical collector by closeloop and open-loop motor control. The chart shows little difference between the close-loop and open-loop motor controls, so their piezoelectric properties were almost the same.

From Fourier transform infrared (FTIR) spectroscopy, Fig. 10 spectra reveal that the fraction of $\beta$-phases $\left(475,510,840,1234,1275\right.$, and $\left.1431 \mathrm{~cm}^{-1}\right)$ with piezoelectric properties increased after

(a)

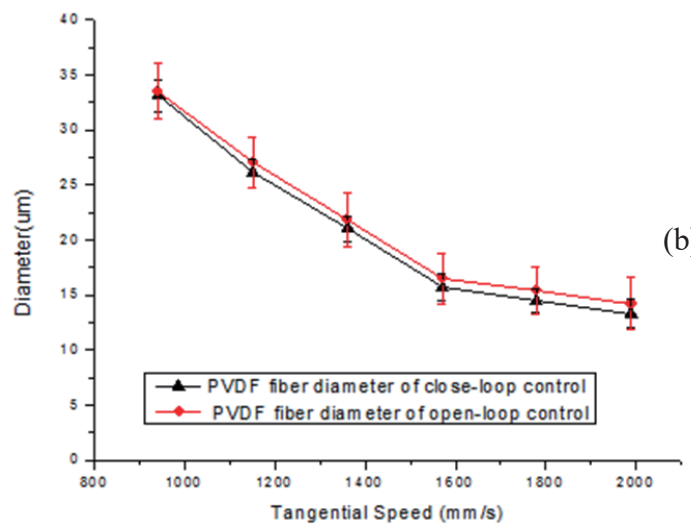

Fig. 7. (Color online) Diameters of PVDF fibers plotted with respect to (a) tangential speed of cylindrical collector and (b) electric field.

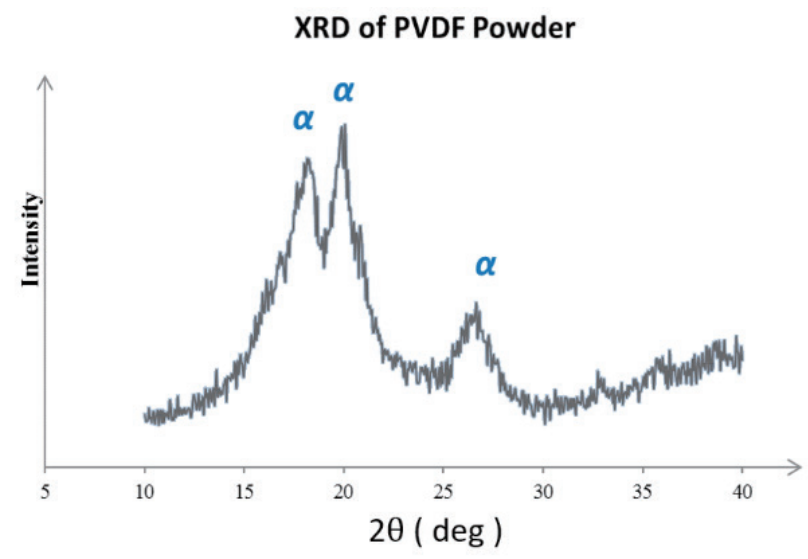

Fig. 8. (Color online) XRD measured at room temperature: PVDF powders before NFES.

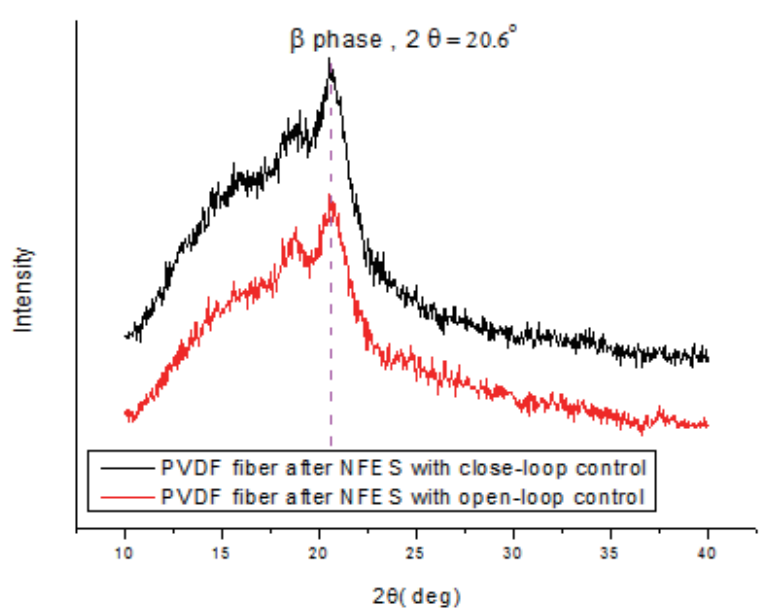

Fig. 9. (Color online) XRD measured at room temperature: PVDF fibers after NFES with close-loop and open-loop motor control in cylindrical collectors. 
the polarization of NFES processing, while the fraction of $\alpha$-phase $(489,531,613,762,795,853$, 970,1209 , and $1382 \mathrm{~cm}^{-1}$ ) decreased.

We used a load cell (brand name: $\mathrm{CONCH}$ ), a microtensile testing machine, to measure the mechanical characteristics of the PVDF fibers. The close-loop motor control for a cylindrical collector had a higher yield point of about $24 \mathrm{MPa}$, and the open-loop motor control for cylindrical collector had a lower yield point of about $20 \mathrm{MPa}$. Their stress-strain diagram is shown in Fig. 11. The open-loop motor control impacted the cylindrical collector speed and the uniformity of the fibers' diameter, thereby resulting in a lower yield point.

We examined the relationship between the voltage and the output power via external resistance of the PVDF fiber array using a close-loop motor control system. The harvesting power can be calculated using Eqs. (1) and (2) (Ohm's law). Then harvesting power can be expressed as Eq. (3):

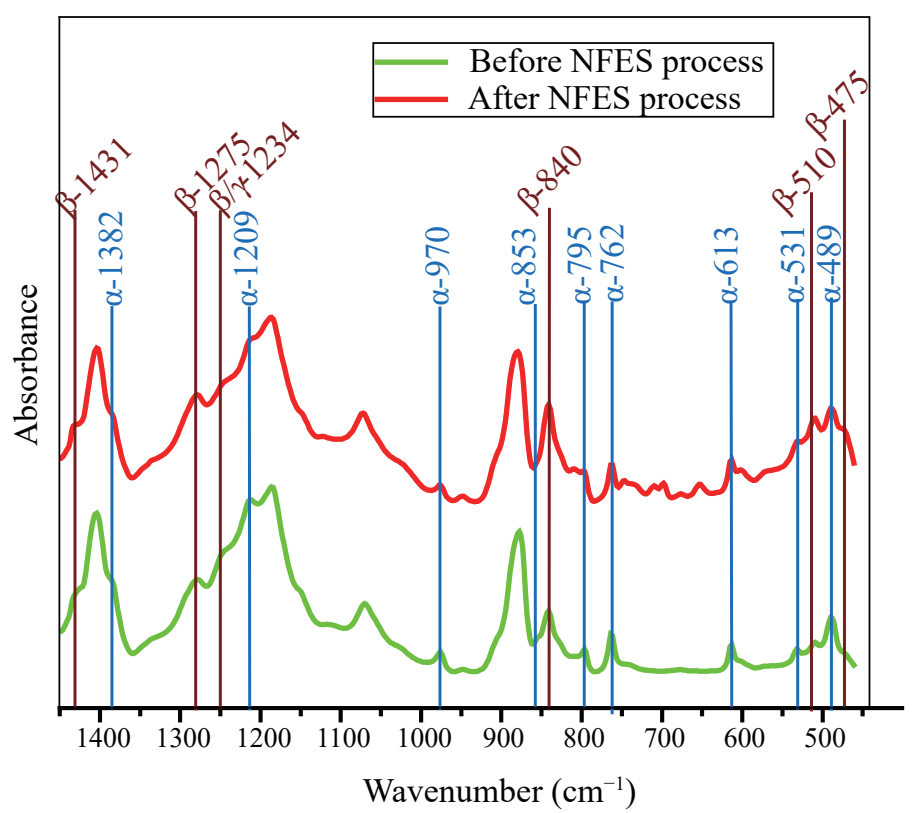

Fig. 10. (Color online) FTIR spectra of PVDF, before and after NFES processing, recorded at room temperature.

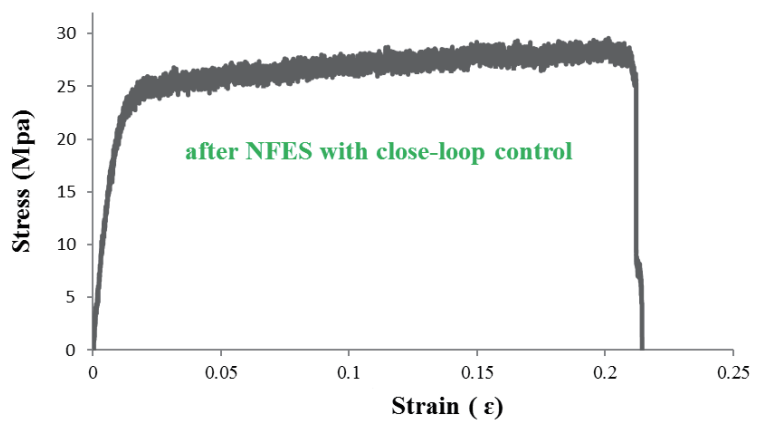

(a)

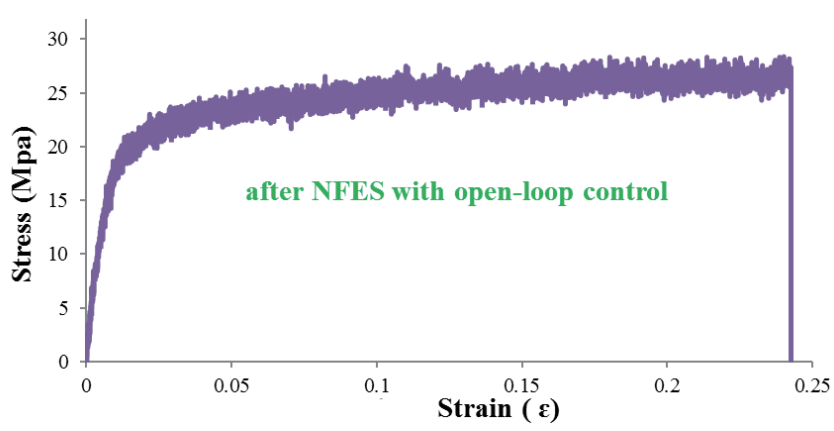

(b)

Fig. 11. (Color online) Stress-strain diagram of PVDF fibers after NFES with (a) close-loop motor control and (b) open-loop motor control in cylindrical collectors. 


$$
\begin{gathered}
P=V_{i}, \\
V=i R, \\
P=V^{2} / R,
\end{gathered}
$$

where $V$ and $i$ are measured voltage and current with an external load resistor, respectively, and $R$ is the external load resistor.

The maximum output power of the PVDF fiber was $427.73 \mathrm{pW}$ when the external resistance was 5.3 $\mathrm{M} \Omega$ and the voltage was $28.13 \mathrm{mV}$ as shown in Fig. 12.

Figure 13 shows SEM images of the close-loop control system [Fig. 13(a)] and open-loop system [Fig.13(b)]. In comparison with the PVDF fibers in Figs. 13(a) and 13(b), the close-loop control fibers show uniform spacing distances and diameters. It makes sense that the close-loop control achieved uniform spacing distances for a stable cylindrical collector speed, because the

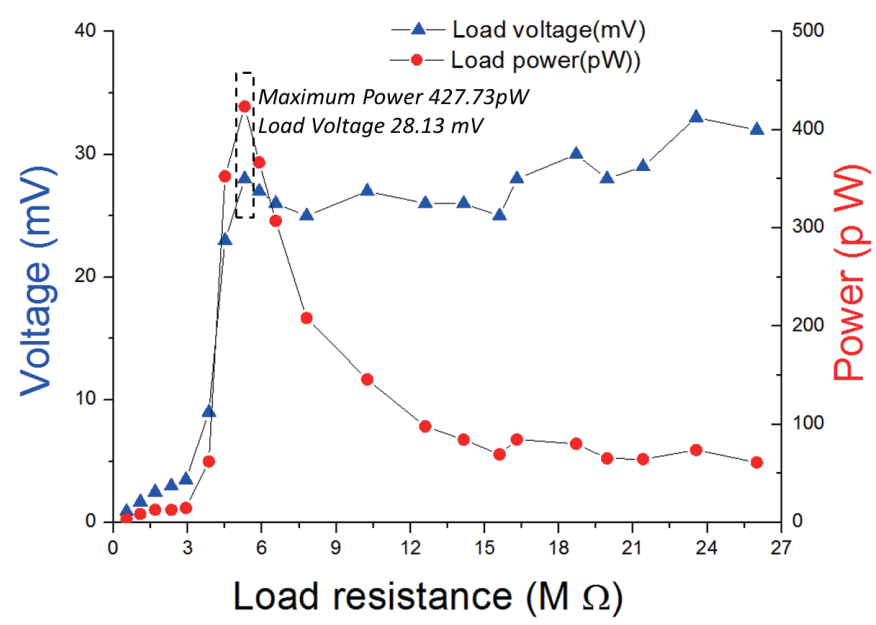

Fig. 12. (Color online) Voltage and output power charts of close-loop control (carried out with parameters of experiment 2 in Table 1).

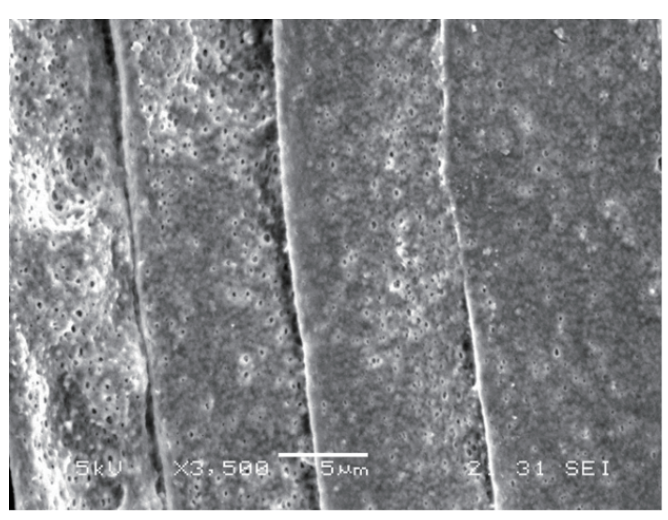

(a)

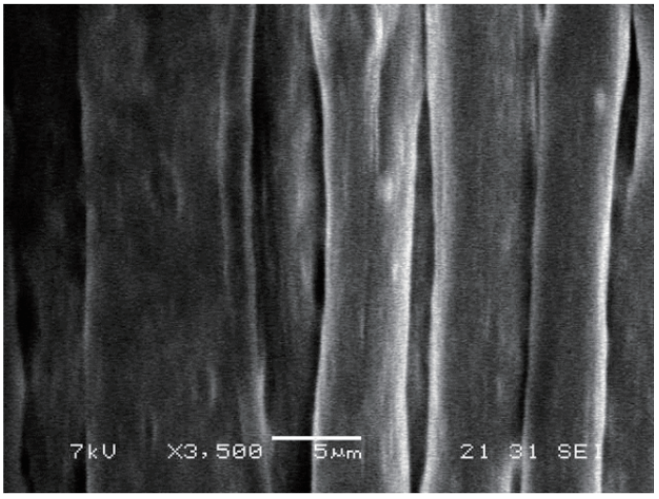

(b)

Fig. 13. (Color online) SEM images obtained with parameters of experiment 2 in Table 1: (a) close-loop motor control system and (b) open-loop system. 
(a)

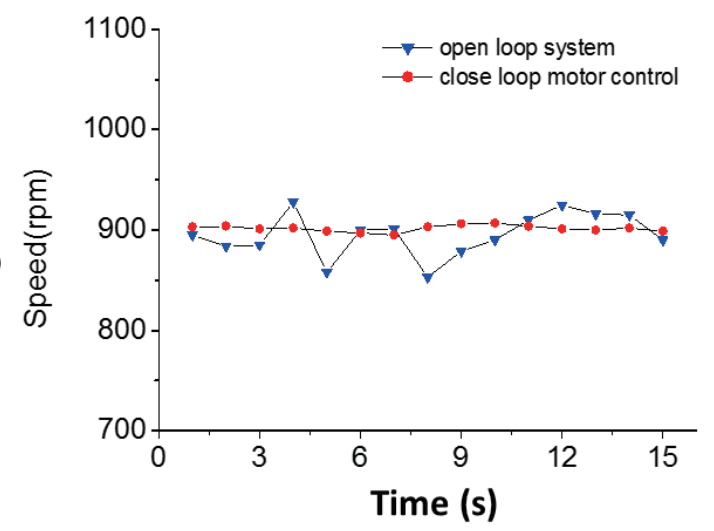

(b)

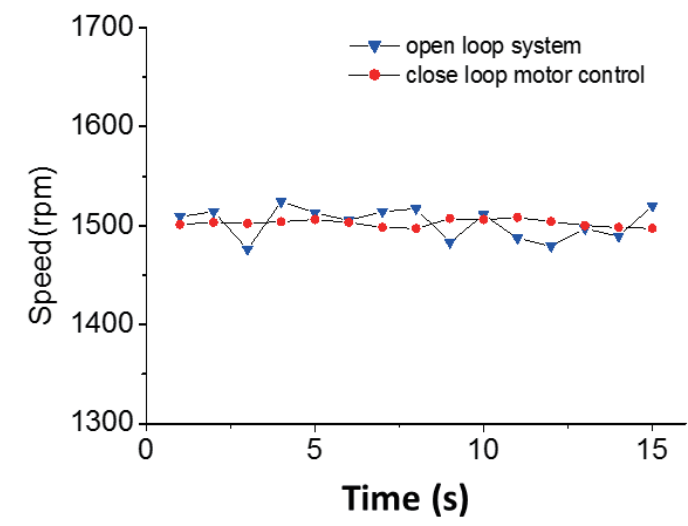

(c)

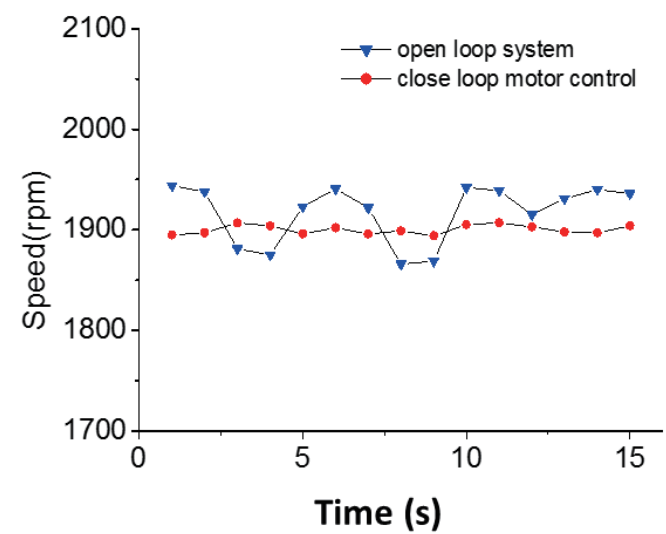

Fig. 14. (Color online) Cylindrical collector speed charts carried out with parameters of experiment 2 in Table 1: close-loop motor control system and open-loop system at (a) 900, (b) 1500, and (c) $1900 \mathrm{rpm}$.

needle travelling speed was constant. A fluctuating cylindrical collector speed resulted in various spacing distances.

Figure 14 shows the comparisons of cylindrical collector speeds at 900, 1500, and $1900 \mathrm{rpm}$, between the close-loop control and the open-loop system. The speed of the cylindrical collector was maintained with the desired speed commands in the close-loop system during fiber collection, but the speed fluctuated in the open-loop control. The close-loop system had the clear advantage of greater accuracy over the open-loop system. Therefore, a constant force resulted in uniform diameters, whereas a fluctuating force resulted in variation in diameters.

\section{Conclusions}

In summary, in this study, we performed experiments with NFES to fabricate continuous PVDF piezoelectric fiber arrays. The experimental scheme was designed using Taguchi Methods, which included five factors with 6 and 3 levels. The close-loop system for motor speed control kept the cylindrical collector at an accurate and steady rotation speed to carry out the $\mathrm{L}_{18}\left(6^{1} \times 3^{6}\right)$ orthogonal array experiments, as shown in Table 1. Comparison of the voltage and current in close-loop and open-loop systems clearly showed that the close-loop system is superior to the open-loop system. The morphology from SEM photos also provided another perspective for their comparison. In 
the case of experiment 2 with $\mathrm{L}_{18}\left(6^{1} \times 3^{6}\right)$ orthogonal arrays were carried out using the close-loop control and open-loop system. After electric property measurements, the PVDF piezoelectric fiber array with close-loop control achieved about $I_{p-p}=8.8 \times 10^{-7} \mathrm{~A}\left(I_{\max }=-5.0 \times 10^{-7} \mathrm{~A}\right)$ and $V_{p-p}=$ $0.22 \mathrm{~V}\left(V_{\max }=-0.12 \mathrm{~V}\right)$; the PVDF piezoelectric fiber array with the open-loop system achieved about $I_{p-p}=3.2 \times 10^{-7} \mathrm{~A}\left(I_{\max }=-2.1 \times 10^{-7} \mathrm{~A}\right)$ and $V_{p-p}=0.17 \mathrm{~V}\left(V_{\max }=-0.10 \mathrm{~V}\right)$. The SEM images also provided morphological evidence showing better uniform spacing and diameters of PVDF fibers with the close-loop control system. Therefore, in this study, we demonstrated the clear improvement with the close-loop motor speed control system compared with open-loop system.

\section{Author Contributions}

Dr. Pan proposed the concept of the experiment. K. C. Tsai planned and executed the experiment, then performed data analyses and wrote the article. Dr. Sun set up the control system equipment and wrote the control system part of the manuscript. C. K. Yen set up the other experiment equipment and performed some data analyses.

\section{Conflicts of Interest}

The authors declare no conflicts of interest.

\section{References}

1 J. Chang, M. Dommer, C. Chang, and L. Lin: Nano Energy 1 (2012) 356.

2 Z. H. Liu, C. T. Pan, C. Y. Su, L. W. Lin, Y. J. Chen, and J. S. Tsai: Sens. Actuators, A 211 (2014) 78.

3 J.-Q. Liu, H.-B. Fang, Z.-Y. Xu, X.-H. Mao, X.-C. Shen, D. Chen, H. Liao, and B.-C. Cai: Microelectron. J. 39 (2008) 802.

4 H.-B. Fang, J.-Q. Liu, Z.-Y. Xu, L. Dong, L. Wang, D. Chen, B.-C. Cai, and Y. Liu: Microelectron. J. 37 (2006) 1280.

5 C. T. Pan, Z. H. Liu, Y. C. Chen, and C. F. Liu: Sens. Actuators, A 159 (2010) 96.

6 C. T. Pan, Z. H. Liu, and Y. C. Chen: Curr. Appl. Phys. 12 (2012) 684.

7 X. Wang: Nano Energy 1 (2012) 13.

8 S. Roundy, E. S. Leland, J. Baker, E. Carleton, E. Reilly, E. Lai, B. Otis, J. M. Rabaey, V. Sundararajan, and P. K. Wright: IEEE Pervasive Comput. 4 (2005) 28.

9 J. M. Donelan, Q. Li, V. Naing, J. A. Hoffer, D. J. Weber, and A. D. Kuo: Science 319 (2008) 807.

10 J. Granstrom, J. Feenstra, H. A. Sodano, and K. Farinholt: Smart Mater. Struct. 16 (2007) 1810.

11 S. Priya: J. Electroceram. 19 (2007) 167.

12 A. P. Chandrakasan, N. Verma, and D. C. Daly: Annu. Rev. Biomed. Eng. 10 (2008) 247.

13 C. Chang, K. Limkrailassiri, and L. Lin: Appl. Phys. Lett. 93 (2008) 123111.

14 I. S. Yeo, J. E. Oh, L. Jeong, T. S. Lee, S. J. Lee, W. H. Park, and B. M. Min: Biomacromolecules 9 (2008) 1106.

15 D. Sun, C. Chang, S. Li, and L. Lin: Nano Lett. 6 (2006) 839.

16 C. Chang, V. H. Tran, J. Wang, Y. K. Fuh, and L. Lin: Nano Lett. 10 (2010) 726.

17 J. Pu, X. Yan, Y. Jiang, C. Chang, and L. Lin: Sens. Actuators, A 164 (2010) 131.

18 H. Kawai: Japanese J. Appl. Phys. 8 (1969) 975.

19 A. G. Holmes-Siedle, P. D. Wilson, and A. P. Verrall: Mater. Design 4 (1983) 910.

20 C. K. Lee: J. Acoust. Soc. Am. 90 (1991) 945.

21 A. Spineanu, P. Bénabès, and R. Kielbasa: Sens. Actuators, A 60 (1997) 127.

22 Z. Chen, Y. Shen, N. Xi, and X. Tan: Smart Mater. Struct. 16 (2007) S262.

23 Y.-S. Lee, S. J. Elliott, and P. Gardonio: Smart Mater. Struct. 12 (2003) 541.

24 C.-T. Pan, C.-K. Yen, S.-Y. Wang, Y.-C. Lai, L. Lin, J. C. Huang, and S.-W. Kuo: RSC Adv. 5 (2015) 85073. 